\title{
The Effect of Boss Cap Fins to B - Series Propeller Performance With CFD Method
}

\author{
Amiadji, Achmad Baidowi, Achdri Fauzi Nugraha Oloan
}

\begin{abstract}
In order to increase propeller performance, Engineer is developing Energy Saving Devices ( ESD ) to reduces operational cost, clean energy and for long term utilization. The global emmision from marine is $2.7 \%$ in 2007. One of ESD is Propeller Boss Cap Fins (PBCF ). The focus of this thesis is to design and developed PBCF B - series propeller. This thesis studies the performance of a propeller without and with PBCF such as efficiency, thrust, torsion and dynamic hub vortex phenomenon. To obtain the results this thesis uses Computational Fluid Dynamic (CFD). The PBCF simulations were based on its pitch angle. The simulation results shows that $P B C F$ is achieved the highest efficiency which is $0.60 \%$, and increases thrust $3.21 \%$, and torsion increase $2.64 \%$ compared to propeller without $P B C F$. It also shows that the PBCF is able to break the vortex flow and it will reduces the porosity to the rudder and decrese the corrosion potention to the rudder.
\end{abstract}

Keywords-CFD, hub vortex PBCF, Thrust, Torsion

\section{INTRODUCTION}

A ccording to (IMO) Internasional Maritime Organization, global emision about $2.7 \%$ from marine activity at 2007[2]. For ESD commontly devide into 8 devices. They are : Twisted Rudder, Costa - Bulb with Twisted Rudde, Costa - Bulb with Conventional Rudder, Boss Cap Fins, Propeller Optimizing, Wake Equalising Duct, Becker Mewis Duct, Bullbous Bow Retro - Fit. From that, its devices have different characteriztic and efficiency [9].

Since developed in 1987 by Mitsui O.S.K Lines, West Japan Fluid Engineering Laboratory, and Nakashima Mitsuwa Propeller was adapted over more than 2000 vessel at world wide [11]. The next stage of the development was conducted by Ouchi [4], since that there are several researches regarding the PBCF. This paper focuses on analyze increases performance of propeller including thrust, torsion, efficiency and eliminating hub vortex which reduces the propeller efficiency and may caused rudder corrotion [7] behind the propeller after and before installing PBCF.

\section{METHOD}

This research using experimental method and comparative method. Experimental method is study of cause, effect and it differs from non - experimental method in that involver the deliberate manipulation of one variable while trying to keep all other variables constant [10]. Comparative method for this research because the author who make compared result before and after propeller performance caused by installation PBCF. It will try to make new design of PBCF according shape and hub fortex phenomenon whic affect the propeller. performance. The role of the fins is weaken energi from rotating flow around propeller cone and the fins can impact of increase propeller efficiency [1].

\section{A. Propeller Modeling}

Propeller modelling using (CAD) Computer Aided Design Software. It can be making geometry and send to the (CFD) Computer Fluid Dynamic Software for take the data. For detail can shown in Figure. 1.

\section{B. PBCF Modeling With Pitch Angle}

PBCF design depend on fluid flow and hub vortex of Bseries propeler. This paper propose a NACA foil as the blade with pitch angle $70^{\circ}$.

\section{Flow Simulation, Thrust and Momment}

Flow simulation for this thesis are using (CFD) Computational Fluid Dynamic Software. From that method, we can find thust and momment propeller Bseries with or without PBCF. Each analyse, can determine from each $(\mathrm{J})$ advance velocities.

\section{RESULTS AND DISSCUSSION}

\section{A. Propeller Boss Cap Fins Geometry}

The propeller model was build in CAD Software. PBCF geometry was built based on NACA foil and in half circle blade. The position of the PBCF blade based on the flow behind the main propeller. The main propeller for this paper uses B4-85 which can be shown in Table. 1.

The specification of the PBCF can be shown in Table. 2. Where the PBCF has the same number of blade as the propeller [8].

\section{B. Mesh Generation}

In order the solver manager to solve the computation based on RANS. The model which has been built in 3D model is meshed in full hexahedral unstructured meshes. The model geometry built into the object and boundary

\footnotetext{
Amiadji, Departement of Marine Engineering, Institut Teknologi Sepuluh Nopember, Surabaya 60111, Indonesia, E-mail: amiadji@yahoo.com. Achmad Baidowi, Departement of Marine Engineering, Institut Teknologi Sepuluh Nopember, Surabaya 60111, Indonesia, E-mail: ahmadbai@gmail.com.

Achdri Fauzi Nugraha Oloan, Departement of Marine Engineering, Institut Teknologi Sepuluh Nopember, Surabaya 60111, Indonesia, E-mail: fauzioji@gmail.com.
} 
condition which represents the environment around the object as can be shown in Figure. 2. The object is combination of trailing edge, leading edge, tip and hub of the system. The inlet and outlet built and meshed also.

The meshing result for the propeller is $2,604,431$ cell and 2,844,566 vertices while the propeller installed with $\mathrm{PBCF}$ is $3,279,982$ cells and 3,593,238 vertices as shown in Table. 3.

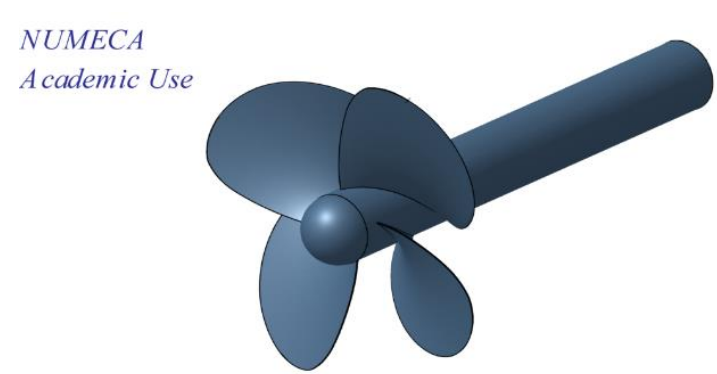

NUMECA

Academic Use

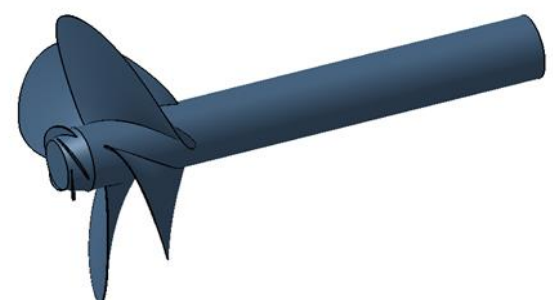

Figure. 1. (Left) Propeller B4-85, (Right) propeller B4-85 with PBCF

TABLE. 1.

SPESIFICATION B4 - 85 PROPELLER

\begin{tabular}{ccccc}
\hline \multicolumn{3}{c}{ SPESIFICATION B4-85 PROPELLER } \\
\hline 1. & $\begin{array}{c}\text { Diameter } \\
(\mathrm{m})\end{array}$ & $\begin{array}{c}\text { Number } \\
\text { Blade }\end{array}$ & $\begin{array}{c}\text { Propeller Prinsipal Dimention } \\
\text { Rotation } \\
(\text { Direction) }\end{array}$ & $\begin{array}{c}\text { Revolution } \\
(\text { Rpm })\end{array}$ \\
\hline 1.262 & 4 & Left & 210 \\
\hline
\end{tabular}

TABLE. 2.

SPESIFICATION PROPELLER Boss CAP Fins

\begin{tabular}{|c|c|c|c|c|}
\hline \multirow[b]{2}{*}{ No } & \multirow[b]{2}{*}{$\begin{array}{l}\text { Diameter } \\
(\mathrm{m})\end{array}$} & \multirow[b]{2}{*}{$\begin{array}{c}\text { Number } \\
\text { Blade }\end{array}$} & \multicolumn{2}{|c|}{$\begin{array}{c}\text { Propeller Boss Cap Fins Principa } \\
\text { Dimention }\end{array}$} \\
\hline & & & $\begin{array}{l}\text { Rotation } \\
\text { (Direction) }\end{array}$ & $\begin{array}{l}\text { Revolution } \\
\text { (Rpm) }\end{array}$ \\
\hline 1. & 0.816 & 4 & Left & 210 \\
\hline
\end{tabular}

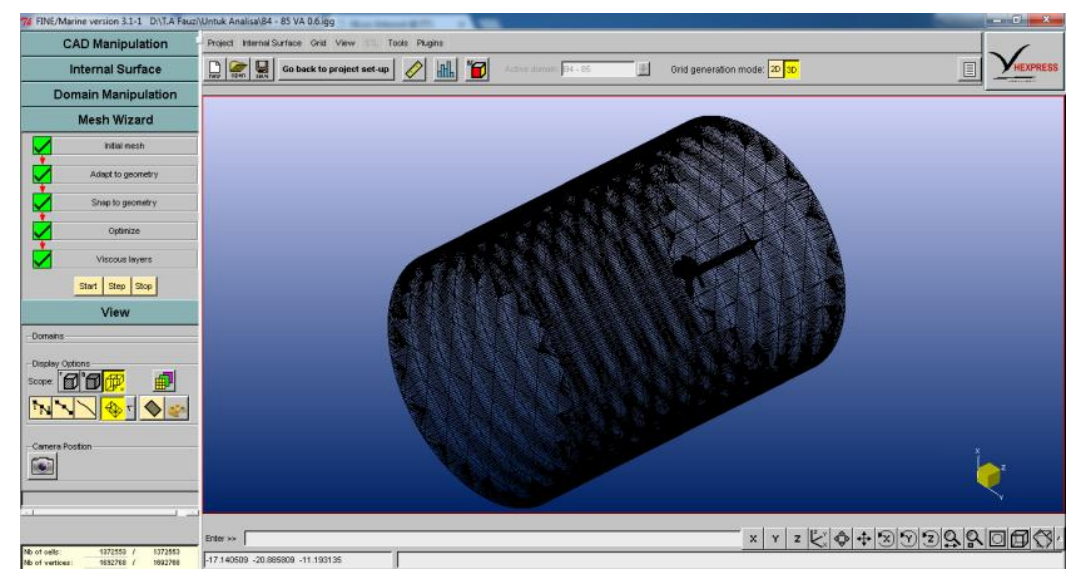

Figure. 2. Meshing Geometry Propeller B4 - 85

TABLE. 3.

MESH QuALITY DETAIL

\begin{tabular}{cccc}
\multicolumn{3}{c}{ MesH QuALITY DETAIL } \\
\hline No & Model & $\begin{array}{c}\text { Total } \\
\text { Number } \\
\text { of Cells }\end{array}$ & $\begin{array}{c}\text { Mesh Quality } \\
\text { Total Number of } \\
\text { Vertices }\end{array}$ \\
\hline 1. & $\begin{array}{c}\text { Propeller } \\
\text { B series. }\end{array}$ & $2,604,431$ & $2,844,566$ \\
2. & $\begin{array}{c}\text { Propeller } \\
\text { With } \\
\text { PBCF }\end{array}$ & $3,279,982$ & $3,593,238$ \\
\hline
\end{tabular}


C. Flow Setting

Flow setting is to define the type of physical configuration of the flow such as k- $\omega$ SST for turbulence model. The cylinder of the free slip domain was applied and the propeller or the object is using non slip wall. The domain setting is $3 \mathrm{D}$ in radius and $5 \mathrm{D}$ in length of the cylinder.

The cavitation which enable the hub vortex to be simulated is activated.

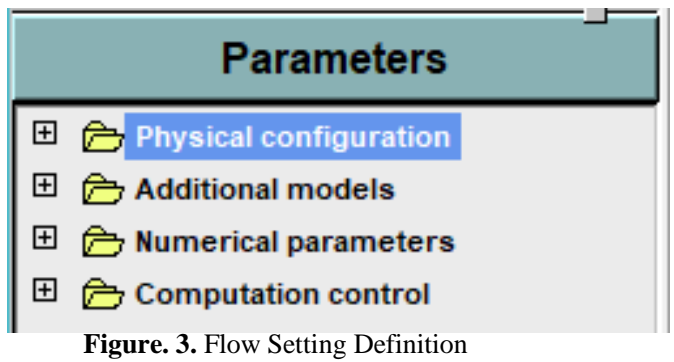

\section{Post Processing}

This final Step is post processing. For CFD. Result from flow setting that analyze was implemented to vector, pressure, turbulency depend from output value that we design.

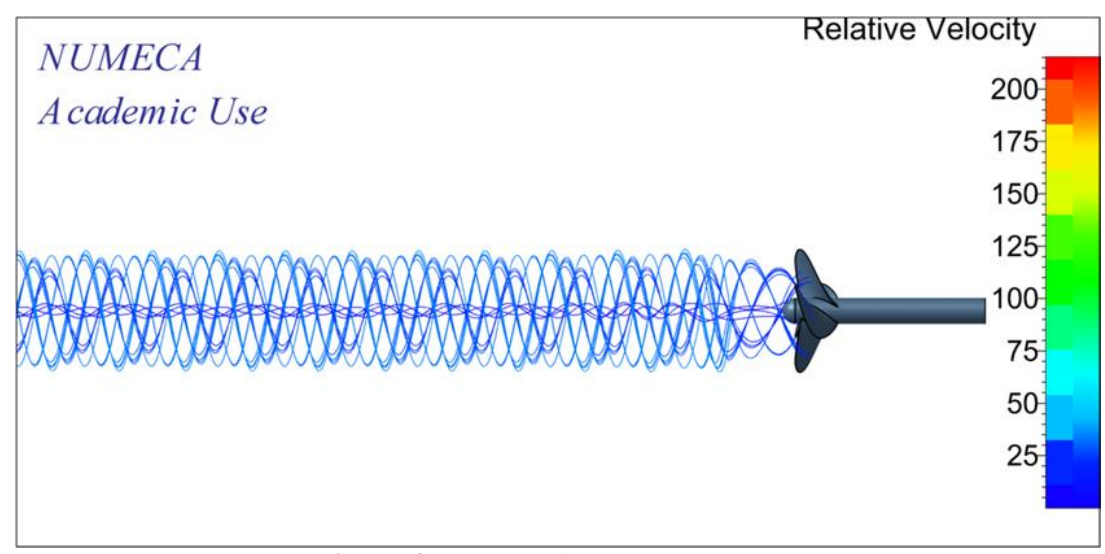

Figure. 4. Example of Post Processing Result

\section{E. Validation of Thrust and Momment From Theory} vs $C F D$
Before install PBCF to propeller B-Series, it must be validate from manual calculation result vs $C F D$ result.

TABLE. 4

Manual CAlculation PROPEller B-SeRies

\begin{tabular}{|c|c|c|c|c|c|c|}
\hline \multicolumn{7}{|c|}{ Result of Manual Calculation } \\
\hline No & $\mathrm{J}$ & KT & $10 * \mathrm{KQ}$ & Efficiency & $\begin{array}{l}\text { Trust } \\
(\mathrm{kN})\end{array}$ & $\begin{array}{c}\text { Momment } \\
(\mathrm{kNm})\end{array}$ \\
\hline 1. & 0.100 & 0.354 & 0.468 & 0.120 & 503.268 & 217.033 \\
\hline 2. & 0.200 & 0.320 & 0.428 & 0.238 & 454.931 & 198.483 \\
\hline 3. & 0.300 & 0.274 & 0.384 & 0.341 & 389.535 & 178.075 \\
\hline 4. & 0.400 & 0.230 & 0.330 & 0.444 & 326.982 & 153.036 \\
\hline 5. & 0.500 & 0.191 & 0.276 & 0.551 & 271.537 & 127.994 \\
\hline 6. & 0.600 & 0.144 & 0.225 & 0.612 & 205.003 & 104.343 \\
\hline 7. & 0.700 & 0.093 & 0.175 & 0.589 & 131.504 & 81.155 \\
\hline 8. & 0.800 & 0.040 & 0.125 & 0.408 & 56.866 & 57.968 \\
\hline
\end{tabular}

CFD RESULT OF PROPELLER B-SERIES

Result of Computational Fluid

Dynamic

\begin{tabular}{ccccccc} 
No & J & KT & $10 *$ KQ & Efficiency & $\begin{array}{c}\text { Trust } \\
(\mathrm{kN})\end{array}$ & $\begin{array}{c}\text { Momment } \\
(\mathrm{kNm})\end{array}$ \\
\hline 1. & 0.100 & 0.305 & 0.405 & 0.120 & 433.252 & 187.774 \\
2. & 0.200 & 0.291 & 0.392 & 0.237 & 413.460 & 181.564 \\
3. & 0.300 & 0.262 & 0.361 & 0.346 & 371.775 & 167.275 \\
4. & 0.400 & 0.227 & 0.324 & 0.446 & 322.804 & 150.224 \\
5. & 0.500 & 0.191 & 0.283 & 0.537 & 270.994 & 131.064 \\
6. & 0.600 & 0.149 & 0.237 & 0.599 & 211.736 & 110.122 \\
7. & 0.700 & 0.107 & 0.189 & 0.628 & 151.498 & 87.737 \\
8. & 0.800 & 0.063 & 0.138 & 0.585 & 89.855 & 63.811 \\
\hline
\end{tabular}


TABLE. 6.

DEVIATION MANUAL VS CFD RESUlT OF B-SERIES

\begin{tabular}{ccccc}
\hline \multicolumn{5}{c}{ Deviation Between Manual } \\
\multicolumn{5}{c}{ Calculation Vs CFD } \\
No & $\mathrm{J}$ & $\begin{array}{c}\Delta \text { KT } \\
(\%)\end{array}$ & $\begin{array}{c}\Delta 10 * \mathrm{KQ} \\
(\%)\end{array}$ & $\begin{array}{c}\Delta \text { Efficiency } \\
(\%)\end{array}$ \\
\hline 1. & 0.100 & 16.16 & 15.58 & 0.50 \\
2. & 0.200 & 10.03 & 9.32 & 0.65 \\
3. & 0.300 & 4.78 & 6.46 & 1.58 \\
4. & 0.400 & 1.29 & 1.87 & 0.57 \\
5. & 0.500 & 0.20 & 2.34 & 2.60 \\
6. & 0.600 & 3.18 & 5.25 & 2.18 \\
7. & 0.700 & 13.20 & 7.50 & 6.16 \\
8. & 0.800 & 36.71 & 9.16 & 30.33 \\
\hline
\end{tabular}

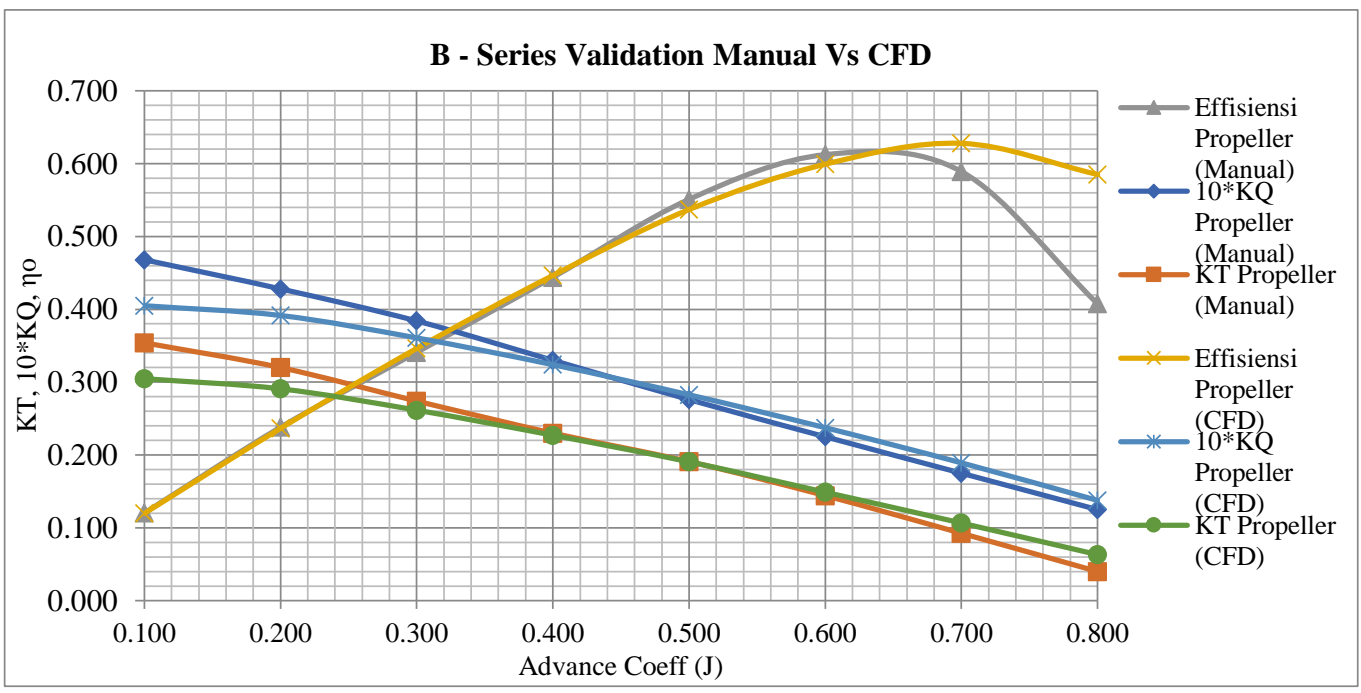

Figure. 5. Open Water Test Validation Resul

From Table 4 we can conclude the validation model for propeller B4-85, validation do by each $(\mathrm{J})$ velocity advance. The deviation very difference each J. For the detail can see figure 5 that represents deviation from $\mathrm{J}$ $=0.1$ to $\mathrm{J}=0.9$. From this model, the maximum deviation at $\mathrm{J}=0.8$ and minimum deviation at $\mathrm{J}=0.4$.
F. PBCF Installation effect on Propeller B-Series After the data has been obtained, difference between before and after installation $\mathrm{PBCF}$ will provide performance improvement. The detail can seen Table 5

TABLE. 7.

RESULT OF WITHOUT PBCF

\begin{tabular}{|c|c|c|c|c|c|c|}
\hline \multicolumn{7}{|c|}{ Without PBCF } \\
\hline No & $\mathrm{J}$ & KT & $10 * \mathrm{KQ}$ & Efficiency & $\begin{array}{l}\text { Trust } \\
(\mathrm{kN})\end{array}$ & $\begin{array}{c}\text { Momment } \\
(\mathrm{kNm})\end{array}$ \\
\hline 1. & 0.100 & 0.305 & 0.405 & 0.120 & 433.252 & 187.774 \\
\hline 2. & 0.200 & 0.291 & 0.392 & 0.237 & 413.460 & 181.564 \\
\hline 3. & 0.300 & 0.262 & 0.361 & 0.346 & 371.775 & 167.275 \\
\hline 4. & 0.400 & 0.227 & 0.324 & 0.446 & 322.804 & 150.224 \\
\hline 5. & 0.500 & 0.191 & 0.283 & 0.537 & 270.994 & 131.064 \\
\hline 6. & 0.600 & 0.149 & 0.237 & 0.599 & 211.736 & 110.122 \\
\hline 7. & 0.700 & 0.107 & 0.189 & 0.628 & 151.498 & 87.737 \\
\hline 8. & 0.800 & 0.063 & 0.138 & 0.585 & 89.855 & 63.811 \\
\hline \multicolumn{7}{|c|}{$\begin{array}{c}\text { TABLE. } 8 . \\
\text { RESULT OF WITH PBCF }\end{array}$} \\
\hline \multicolumn{7}{|c|}{ With PBCF } \\
\hline No & $\mathrm{J}$ & KT & $10 * \mathrm{KQ}$ & Efficiency & $\begin{array}{l}\text { Trust } \\
(\mathrm{kN})\end{array}$ & $\begin{array}{l}\text { Momment } \\
(\mathrm{kNm})\end{array}$ \\
\hline 1. & 0.100 & 0.306 & 0.409 & 0.120 & 437.513 & 189.620 \\
\hline 2. & 0.200 & 0.294 & 0.396 & 0.237 & 418.000 & 183.481 \\
\hline 3. & 0.300 & 0.267 & 0.366 & 0.348 & 379.207 & 169.958 \\
\hline 4. & 0.400 & 0.233 & 0.330 & 0.450 & 331.408 & 153.175 \\
\hline 5. & 0.500 & 0.195 & 0.289 & 0.537 & 276.830 & 133.318 \\
\hline 6. & 0.600 & 0.154 & 0.244 & 0.602 & 218.800 & 113.318 \\
\hline 7. & 0.700 & 0.112 & 0.197 & 0.633 & 159.307 & 91.578 \\
\hline 8. & 0.800 & 0.069 & 0.147 & 0.601 & 96.553 & 68.188 \\
\hline
\end{tabular}


TABLE. 9. INCREASEMENT RESULT

\begin{tabular}{ccccc}
\hline \multicolumn{5}{c}{ Increasement Performance } \\
No & $\mathrm{J}$ & $\begin{array}{c}\Delta \text { Trust } \\
(\%)\end{array}$ & $\begin{array}{c}\Delta \text {,Mommert } \\
(\%)\end{array}$ & $\begin{array}{c}\Delta \text { Efficiency } \\
(\%)\end{array}$ \\
\hline 1. & 0.100 & 0.97 & 0.97 & 0.00 \\
2. & 0.200 & 1.09 & 1.04 & 0.04 \\
3. & 0.300 & 1.96 & 1.58 & 0.39 \\
4. & 0.400 & 2.60 & 1.93 & 0.68 \\
5. & 0.500 & 2.11 & 2.17 & 0.07 \\
6. & 0.600 & 3.23 & 2.82 & 0.42 \\
7. & 0.700 & 4.90 & 4.19 & 0.74 \\
8. & 0.800 & 8.83 & 6.42 & 2.57 \\
& Average & 3.21 & 2.64 & 0.60 \\
\hline
\end{tabular}

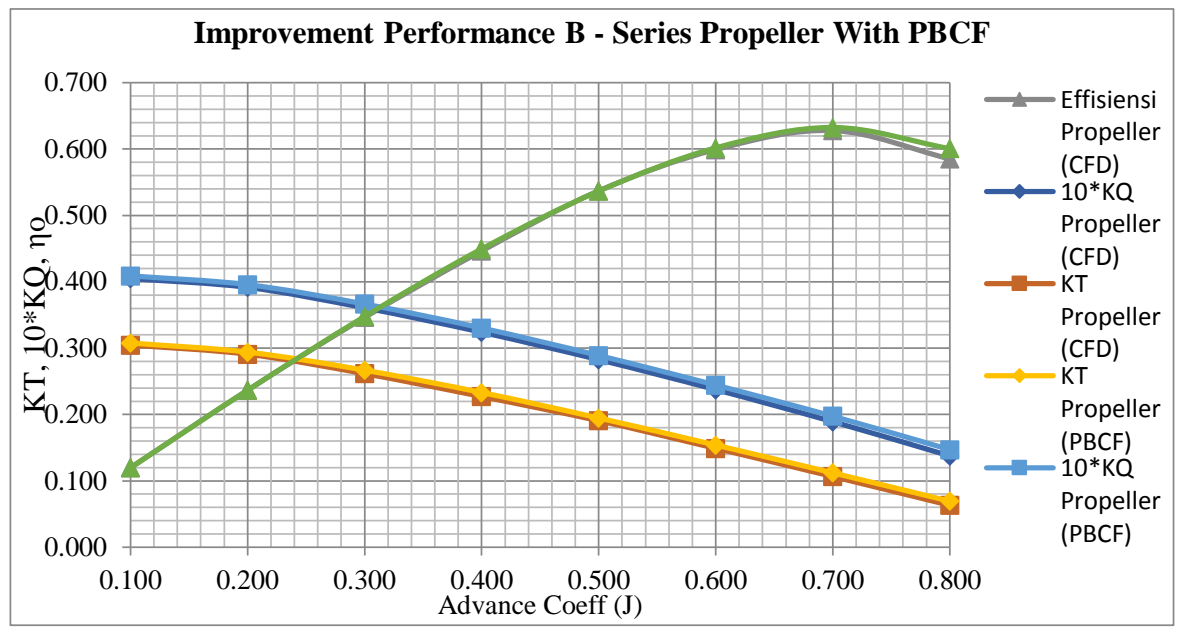

Figure. 6. Open Water Test Propeller Vs PBCF

From Table 5 we can conclude performance between propeller B4-85 vs B4-85 with $\mathrm{PBCF}$, average improvement performance from $\mathrm{J}=0.1$ until $\mathrm{J}=0.8$ is efficiency ( $0.60 \%$ ), Thrust $(3.21 \%$ ) and momment $(2.64 \%)$. Higher efficiency can impact better performance.

G. Fluid Flow Analyze
Make sure if the data are correct, we must check the fluid flow for see the detail of fluid flow and hub vortex phenomenon. Propeller whithout PBCF can be explained by Figure 7. From that we can conclude strong hub vortex generated by propeller itshelf. But figure 8 unformed hub vortex phenomenon because fluid flow from propeller is blocked by fins and caused fluid flow can not forming hub vortex.

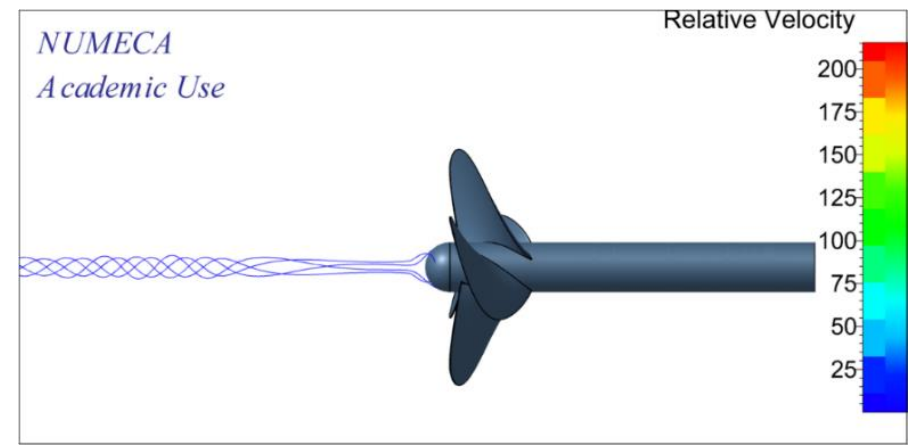

Figure. 7. Strong Hub Vortex Formed at Propeller Whitout PBCF

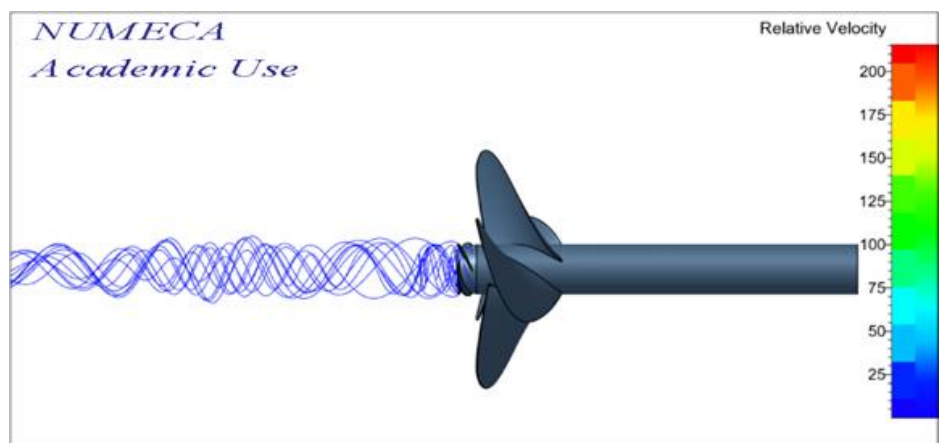

Figure. 8. Unformed Hub Vortex Fortex Propeller Whith PBCF 


\section{CONCLUSIONS}

From that several result of simulation, there are several main conclusions which can be describe bellow :

A.Open water test result B-Series produce average efficiency $0.437 \%$, thrust $283.173 \mathrm{kN}$ and momment $134.946 \mathrm{kNm}$

B. Open water test result obtained from CFD shows that PBCF improve the efficiency around $0.60 \%$, thrust is increased around $3.21 \%$ in average and momment increases $2.64 \%$.

C. $\mathrm{PBCF}$ is able to reduce hub vortex which resulted rudder corrosion reduction and provide additional thrust for the propeller and reducing rudder corrotions.

\section{REFERENCES}

[1] Cartlon, J.S.(2007). Marine Propeller and Propultion, Elsevier, Great Britain

[2] IMO, WMU (2013). Training Course Energi Efficient Operation Of Ships

[3] Babicz Jan (2015). Wartsila Encyclopedia Of Ship Technology,Wartsila, Helsinki

[4] Lianzhou Wang, 2017. Numerical analysis of a propeller during heave motion in cavitation flow, ELSEVIER, Harbin.

[5] MA Cheng, 2014. The design of propeller and propeller boss cap fins (PBCF) by an integrative method, ELSEVIER, Beijing.

[6] Victor N, 2015. Integrated approach to vessel energy efficiency, ELSEVIER, Glasgow.

[7] Kurt Mizzi, 2016. Design optimization of Propeller Boss Cap Fins for enchanced propeller performance, ELSEVIER, Glasgow.

[8] Ouchi,et all., A research and development of PBCF - Improvement of Flow from Propeller Boss, JSNA, Japan.

[9] www.sva-potsdam.de/en/energy-saving-devices/

[10] http://www.holah.karoo.net/experimental_method.htm

[11] PBCF Promotional Video by MOL Techno-Trade,Ltd 